\title{
Manifestaciones cutáneas de lupus eritematoso, esclerosis sistémica y dermatomiositis
}

\author{
Cutaneous manifestations in lupus erythematosus, systemic sclerosis and \\ dermatomyositis
}

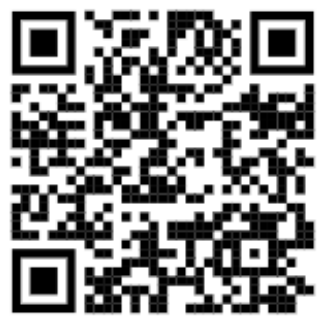

\author{
${ }^{1}$ Dra. Rebeca Zumbado Vásquez \\ Investigadora independiente, Heredia, Costa Rica \\ (D) https://orcid.org/0000-0003-1681-1871 \\ ${ }^{2}$ Dr. Kevin Daniel Hines Chaves \\ Investigador independiente, San José, Costa Rica \\ (D) https://orcid.org/0000-0002-6949-3179 \\ ${ }^{3}$ Dra. Valeria Castro Corrales
} Investigadora independiente, San José, Costa Rica

(D) https://orcid.org/0000-0001-7044-0675

RECIBIDO

$02 / 04 / 2019$

CORREGIDO

ACEPTADO

$16 / 04 / 2019$

$01 / 05 / 2019$

\section{RESUMEN}

Muchas enfermedades reumatológicas se caracterizan por presentar manifestaciones clínicas heterogéneas, que abarcan desde únicamente lesiones cutáneas hasta la afectación de múltiples órganos internos. La mayoría de estos pacientes van a presentar manifestaciones dermatológicas y estas pueden ser las primeras señales de la enfermedad. Identificar las lesiones cutáneas de estas patologías será de utilidad para lograr un diagnóstico y un control óptimo temprano. En esta revisión se abarcarán tres grandes enfermedades reumatológicas: lupus eritematoso, esclerosis sistémica y dermatomiositis.

${ }^{1}$ Médico general, graduada de la Universidad de Costa Rica (UCR). Código médico: 15865 rebezv@gmail.com.

${ }^{2}$ Médico general, graduado de la Universidad de Costa Rica (UCR). Código médico: 15872 kevin.hines19@gmail.com.

${ }^{3}$ Médico general, graduada de la Universidad de Costa Rica (UCR). Código médico: 15820 valeriacastroco@gmail.com.
PALABRAS CLAVE: enfermedades del colágeno; dermatomiositis; lupus eritematoso sistémico; esclerodermia sistémica.

Many rheumatologic diseases are characterized by having heterogeneous clinical manifestations; these can present solely as cutaneous lesions or affect multiple internal organs. Most of these patients will have dermatologic manifestations, which in many cases will be the first sign of the underlying disease. Identifying the different skin lesions in these diseases will help diagnose and control them early and adequately. This review will focus on three of the main rheumatologic diseases: lupus erythematosus, systemic sclerosis and dermatomyositis. 
KEYWORDS: collagen diseases; dermatomyositis; lupus erythematosus, systemic; scleroderma, systemic.

\section{INTRODUCCIÓN}

El lupus eritematoso, la dermatomiositis y la esclerosis sistémica pertenecen a un grupo de enfermedades reumatológicas complejas las cuales pueden plantar un desafío diagnóstico y terapéutico debido a la participación de múltiples sistemas en su patología (1). A pesar de la gran heterogeneidad en sus manifestaciones, el médico debe ser capaz de reconocer ciertos patrones distintivos que aumentan la sospecha de estas patologías.

Por medio de las lesiones en la piel es posible identificar estas patologías tempranamente, e incluso, pueden ofrecer una estimación de la afectación sistémica, la actividad y el pronóstico de la enfermedad (2); esto tiene un gran impacto en el manejo de estos pacientes considerando que estas patologías generan una disminución en la calidad de vida y en ciertos casos un aumento en la mortalidad $(3,4)$.

En esta revisión se expone desde un punto de vista dermatológico los signos y síntomas distintivos de estas tres enfermedades con el objetivo de proveer al lector hallazgos claves en estas patologías para mejorar la precisión del diagnóstico de forma temprana y de esta manera iniciar oportunamente el tratamiento.

\section{LUPUS ERITEMATOSO}

El lupus eritematoso es una enfermedad autoinmune inflamatoria del tejido conectivo, caracterizada por la presencia de anticuerpos y complejos inmunes debido a una alteración en la tolerancia inmunológica (5). Se puede dividir en la forma sistémica o cutánea (6). Hasta el $85 \%$ de los pacientes presentan lesiones cutáneas, las cuales pueden verse en cualquier estadio de la enfermedad, e incluso preceder las manifestaciones sistémicas en un $25 \%$ de los pacientes $(7,8,9)$. Estas se dividen en manifestaciones específicas de lupus y manifestaciones no específicas. Dentro de las específicas se encuentran los subtipos de lupus eritematoso cutáneo (LC), los cuales se determinan según las lesiones clínicas, su duración, y según hallazgos histológicos y de laboratorio. Dentro de este grupo se tiene: lupus cutáneo agudo, subagudo, crónico (5, 8,9). Las lesiones no específicas pueden estar asociadas a otras enfermedades autoinmunes e incluyen cambios vasculares como livedo racemosa, telangiectasias periungeales, tromboflebitis, vasculitis urticaria, calcinosis cutis, alopecia no cicatrizante, eritema multiforme y fenómeno de Raynaud $(5,8)$.

\section{- Lupus cutáneo agudo}

Este subtipo usualmente se presenta con eritema malar o eritema "en alas de mariposa”, el cual puede ser transitorio, con una duración de días a semanas (6). Este eritema clásicamente es inducido por exposición solar y precede las manifestaciones sistémicas. Usualmente es no cicatrizante sin embargo puede resultar en despigmentación, y en 
ocasiones puede generar dolor y prurito $(5,6,10)$.

Las lesiones comienzan como pequeñas maculas y pápulas eritematosas a nivel centro facial que posteriormente confluyen y se expanden sobre mejillas y el puente nasal, sin embargo, respetan los pliegues naso labiales. Ocasionalmente presentan escamas finas y pueden asociar edema facial. Además, puede presentar erosiones y ulceras en mucosa oral y nasal $(5,10)$.

Cuando el LC agudo es generalizado se presenta el eritema maculopapular con una distribución amplia y simétrica, con compromiso de palmas y pies, sin embargo, respeta la unión metacarpofalángica (5). En ocasiones puede asociar prurito y semejar una reacción medicamentosa, o pueden observarse ulceras y aftas en mucosas, además del adelgazamiento del cabello $(5,10)$.

Se puede observar eritema en la zona periungueal, sobrecrecimiento de la cutícula, y puede afectar el dorso de las manos, por lo que en ocasiones puede confundirse con dermatomiositis, sin embargo, en el LC se respetan las uniones metacarpofalángicas e interfalángicas (10).

\section{- Lupus cutáneo subagudo}

Se observan lesiones maculopapulares eritematosas que se distribuyen de manera simétrica en zonas fotoexpuestas. Las lesiones pueden evolucionar a placas escamosas, papuloescamosas 0 presentar una distribución anular. Las placas anulares pueden confluir, formando lesiones con hipopigmentación central. A diferencia del lupus discoide, en este caso no hay lesiones cicatrizantes $(5,11)$. Este subtipo también suele asociar artralgias y artritis, además $10-15 \%$ de los pacientes desarrollan un lupus eritematoso sistémico leve (6).

\section{- Lupus cutáneo crónico}

En esta subdivisión hay múltiples presentaciones, como por ejemplo el lupus discoide, lupus profundus 0 paniculitis, lupus mucoso, entre otros; siendo el primero la presentación más frecuente de estos (6). El lupus discoide puede ser localizado, presentándose en zonas fotoexpuestas como cara, orejas, o cabeza; o involucrar las zonas superior e inferior al cuello, siendo esta la forma diseminada. Se manifiesta como un parche numular o discoide, eritematoso, bien definido, seguido de hiperqueratosis folicular (5). Al remover la escama se observan tapones de queratina que asemejan una alfombra, a lo que se conoce como signo de alfombra" (6). Las lesiones se van expandiendo con inflamación e hiperpigmentación en la periferia, dejando un centro hipopigmentado, atrófico y cicatrizado con telangiectasias. En el cuero cabelludo puede progresar a una alopecia cicatrizante (5).

Existen dos grupos de criterios para clasificar e identificar el lupus eritematoso, unos propuestos por el Colegio Americano de Reumatología (ACR) en 1982, el cual fue actualizado en 1997, con 11 criterios a considerar; y los criterios del 2012 del "Systemic Lupus International Collaborating Clinics (SLICC)" $(7,11,12)$.

EI SLICC 2012 toma en cuenta 17 criterios, y requiere al menos 4 criterios para hacer el diagnóstico, de los cuales uno debe ser clínico y uno de laboratorio. Dentro de los criterios se incluye: la 
alopecia no cicatrizante, sinovitis, serositis, y úlceras orales o nasales (1113). Por otro lado, el ACR 1997 toma en cuenta el eritema malar, el eritema discoide, fotosensibilidad, úlceras orales o nasales y además criterios de laboratorio (12).

\section{DERMATOMIOSITIS}

Pertenece al grupo de miositis idiopáticas inflamatorias, y se caracteriza por hallazgos cutáneos específicos lo cuales frecuentemente se presentan previo a la manifestación muscular. En la dermatomiositis (DM) existe la afectación simétrica de músculos proximales, además de los hallazgos de laboratorio de elevación de deshidrogenasa láctea, transaminasas y enzimas musculares como creatinin quinasa o aldolasa (8).

En algunos casos de DM solamente se tendrá la presentación cutánea y nunca desarrollan miositis (14). Si después de dos años no se ha presentado afectación muscular se hace el diagnóstico de la variante conocida como Dermatomiositis amiopática, la cual constituye aproximadamente $20 \%$ de los casos $(6,8)$.

Se ha descrito que lesiones cutáneas existentes se pueden exacerbar con la exposición solar, incluso se ha visto que quemaduras solares pueden preceder el inicio de DM, por lo que la protección solar va a ser vital en estos pacientes (8). La dermatomiositis se ha asociado con un mayor riesgo de cáncer, por lo que es importante vigilar por signos que nos sugieran malignidad, especialmente de pulmón, ovario, páncreas y estómago (1). Las manifestaciones cutáneas en esta patología son sumamente importantes, contribuyen al diagnóstico temprano y además son útiles para determinar la actividad de la enfermedad (6). El eritema en heliotropo con edema periorbital es una lesión altamente distintiva de la DM. Es un eritema violáceo, se localiza a nivel mediofacial, sobre todo en párpados superiores, e involucra los pliegues nasolabiales, hallazgo que ayuda a diferenciarlo del eritema malar del LES $(3,8)$.

Otras lesiones cutáneas que se identifican en estos pacientes son la erupción en heliotropo en el cuello (conocido como signo del chal), en tórax, que se manifiesta como el "signo de la V"; y en rodillas y codos. También se pueden observar cambios de poiquilodermatomiositis y calcinosis cutis $(3,8)$.

Las pápulas eritematosas o violáceas en la unión metacarpofalangea y la unión interfalángica proximal son conocidas como pápulas de Gottron, estas pueden presentar una descamación y en ocasiones ulcerarse. $(6,8)$. Incluso se ha reportado que las pápulas de Gottron se presentan en mayor frecuencia que del eritema en heliotropo, en hasta el $70 \%$ de los pacientes, por lo que es considerado por muchos el signo clave en el diagnóstico de DM $(3,6)$. El signo de Gottron corresponde a máculas eritematosas 0 eritematoescamosas, simétricas, en la superficie extensora de las extremidades como codos, rodillas, además de la superficie dorsolateral de la articulación metacarpofalángica (14). Tanto las pápulas de Gottron como el signo de Gottron son consideradas signos patognomónicos de DM $(4,6,8)$.

Estos pacientes suelen presentarse con eritema y descamación acompañado de 
prurito y ardor, sobretodo en la piel de la cabeza. Estos dos síntomas son hallazgos importantes a considerar ya que ayudan a diferenciar a esta patología de LC $(3,8)$.

\section{ESCLEROSIS SISTÉMICA}

La esclerosis sistémica (ES) es una enfermedad de tejido conectivo autoinmune en la cual se desarrolla daño microvascular y producción excesiva de fibrosis. A pesar de ser una enfermedad poco frecuente presenta una alta mortalidad, y sus manifestaciones pueden reducir la calidad de vida. $(8,15,16)$. Debido a esta alta morbimortalidad, la identificación de las lesiones cutáneas genera la posibilidad de hacer un diagnóstico más rápido ya que estos signos son la manifestación más temprana y más característica de la enfermedad. Además, ciertos signos permiten medir la actividad de la enfermedad y predecir la afectación de órganos internos (16).

Se puede clasificar como esclerosis sistémica cutánea limitada o difusa, según el grado de compromiso de la piel (15). Su clasificación es importante ya que la ES cutánea difusa tiene un pronóstico más reservado (16).

En la variante limitada va a existir afectación de la cara y de la piel distal a codos y rodillas, esta progresa lentamente y aumenta el riesgo de hipertensión arterial pulmonar (HTAP). Por otro lado, en la presentación difusa hay compromiso del tronco y de la zona proximal de las extremidades, la piel tensa y pruriginosa son hallazgos tempranos. La ES cutánea evoluciona de manera rápida y puede desarrollar daño a nivel renal y cardiaco y generar enfermedad intersticial pulmonar (ILD, por sus siglas en inglés) $(6,8)$.

En un $95 \%$ de los pacientes la primera manifestación de la enfermedad va a ser el fenómeno de Raynaud, el cual puede preceder la patología como tal en hasta 10 años $(8,17)$. Este se caracteriza por un cambio de coloración trifásico debido a vasoespasmo, inicialmente ocurre una vasoconstricción, seguido de cianosis y finalmente hiperemia, generando los cambios de color blanco-azul-rojo (16). El $50-60 \%$ de los pacientes pueden tener úlceras digitales, que afectan la funcionalidad y la calidad de vida. Debido a la reparación lenta de las ulceras se pueden asociar complicaciones como infección local, osteomielitis, dolor y gangrena $(8,16)$.

Estos pacientes presentan facies típicas que se caracterizan por telangiectasias, microstomía y nariz puntiaguda. La piel alrededor de los labios está esclerosada, lo que dificulta la apertura de la boca y le da a la cara un aspecto de "máscara" (3). El engrosamiento de la piel coincide con el compromiso de órganos internos y confiere un peor pronóstico y mayores disabilidades (2).

El diagnóstico de ES se basa en características clínicas, inmunológicas, y hallazgos a nivel de capilares. El hallazgo del engrosamiento de la piel de los dedos de ambas manos que se extiende de manera proximal a la unión metacarpofalángica otorga un puntaje de 9, lo cual es el puntaje mínimo necesario para hacer el diagnostico. Otras características que se consideran para el diagnóstico son la presencia de lesiones en la punta de los dedos, telangiectasias, HTAP o ILD, fenómeno de Raynaud, anticuerpos relacionados con ES (anticentrómero principalmente en la variante 
limitada, y Anti-topoisomerasa o Scl-70 para la ES cutánea difusa) y capilares anormales en el lecho ungueal (8).

Como se mencionó inicialmente, la meta es lograr un diagnóstico temprano, por lo que la presencia de "banderas rojas" como el hallazgo de dedos edematosos y el fenómeno de Raynaud debe alertar de la posibilidad de ES, e inducir a realizar más estudios para confirmar el diagnóstico $(17,18)$.

\section{CONCLUSIÓN}

A través del artículo se describen los distintos signos y síntomas presentes en el lupus eritematoso, la dermatomiositis y la esclerosis sistémica, caracterizando a cada una de ella por medio de las lesiones cutáneas que presentan. Cada una de las enfermedades descritas puede tener lesiones compartidas con otras patologías inmunológicas, sin embargo, hay hallazgos característicos que orientan y aumentan la sospecha de alguna en particular (5).

En la mayoría de los casos, estas patologías se manifiestan inicialmente con lesiones en piel características, o incluso patognomónicas, las cuales incluso pueden preceder la enfermedad sistémica en meses o años (6). Por este motivo, se concluye que es de suma importancia conocer la presentación cutánea en cada una de ellas, y de esta manera, hacer de la inspección de las lesiones cutáneas una herramienta para aproximarse al diagnóstico y asimismo, utilizarlas como medidas de actividad de la enfermedad.

Se espera que posterior a esta revisión el lector logre identificar con mayor facilidad y seguridad los signos y síntomas cutáneos que orientan al lupus eritematoso, la dermatomiositis y la esclerosis sistémica, y que le proporcione datos claves para diferenciar incluso entre ellos mismos.

\section{REFERENCIAS}

1. Naesström M, Kakol M, Kamkar V, Baranska-Rybak W, Sokolowska-Wojdylo M, Stawczyk M, Nowicki R. A rarity that can lead to a casualty - A retrospective study of 12 cases of Dermatomyositis. British Journal of Medical Practitioners. 2015; 8(3): a822. Disponible en:http://www.bjmp.org/content/rarity-can-leadcasualty-retrospective-study-12-cases-dermatomyositis

2. Kumánovics G, Péntek M, Bae S, Opris D, Khanna D, Furst DE, Czirják L. Assessment of skin involvement in systemic sclerosis. Rheumatology. $2017 \quad 09$ 01;56(suppl_5):v53v66.https://doi.org/10.1093/rheumatology/kex202

3. Fitzpatrick T, Goldsmith L, Wolff K. Dermatología en Medicina General. 8va ed. Nueva York: McGraw-Hill; 2012. p. 1909-1956

4. Mainetti C, Beretta-Piccoli BT, Selmi C. Cutaneous Manifestations of Dermatomyositis: a Comprehensive Review. Clinical Reviews in Allergy \& Immunology. 2017; 53(3): 337-356.https://doi.org/10.1007/s12016017-8652-1

5. Kuhn A, Landmann A. The classification and diagnosis of cutaneous lupus erythematosus. Journal of Autoimmunity. 2014 02; 48-49: 14-19. https://doi.org/10.1016/j.jaut.2014.01.021

6. Fadhel SD, Silva ACD. Lupus Erythematosus, Dermatomyositis and Systemic Sclerosis: Assessment and Differentials from A Dermatologic Point of View. Lupus Erythematosus, Dermatomyositis and Systemic Sclerosis: Assessment and Differentials from A Dermatologic Point of View. Enliven: clinical dermatology 
[Internet]. $2018 \quad$ [citado $2019 \quad$ Mar $\quad 18] ; \quad 4(1): \quad 002 . \quad$ Disponible en:https://www.researchgate.net/publication/326804551 Lupus Erythematosus Dermatomyositis and S ystemic Sclerosis Assessment and Differentials from A Dermatologic Point of View

7. Kuhn A, Bonsmann G, Anders H, Herzer P, Tenbrock K, Schneider M. The Diagnosis and Treatment of Systemic Lupus Erythematosus. Deutsches Aerzteblatt Online. 201506 19; 112(25): 423432. https://doi.org/10.3238/arztebl.2015.0423

8. Kuhn A, Landmann A, Bonsmann G. The skin in autoimmune diseases - Unmet needs. Autoimmunity Reviews. 2016 Oct; 15(10): 948-954. https://doi.org/10.1016/j.autrev.2016.07.013

9. Stannard JN, Kahlenberg JM. Cutaneous lupus erythematosus. Current Opinion in Rheumatology. 2016 09; 28(5): :453-459. https://doi.org/10.1097/bor.0000000000000308

10. Okon L, Werth V. Cutaneous lupus erythematosus: Diagnosis and treatment. Best Practice \& Research Clinical Rheumatology. 2013 06; 27(3): 391-404.https://doi.org/10.1016/i.berh.2013.07.008

11. Tiao J, Feng R, Carr K, Okawa J, Werth VP. Using the American College of Rheumatology (ACR) and Systemic Lupus International Collaborating Clinics (SLICC) criteria to determine the diagnosis of systemic lupus erythematosus (SLE) in patients with subacute cutaneous lupus erythematosus (SCLE). Journal of the American Academy of Dermatology. $2016 \quad 05 ; \quad 74(5)$ : $862-$ 869. https://doi.org/10.1016/j.jaad.2015.12.029

12. Inês L, Silva C, Galindo M, López-Longo FJ, Terroso G, Romão VC, Rúa-Figueroa I, et al. Classification of Systemic Lupus Erythematosus: Systemic Lupus International Collaborating Clinics Versus American College of Rheumatology Criteria. A Comparative Study of 2,055 Patients From a Real-Life, International Systemic Lupus Erythematosus Cohort. Arthritis Care \& Research. 201507 26; 67(8) :11801185. https://doi.org/10.1002/acr.22539

13. Yeoh S, Dias SS, Isenberg DA. Advances in systemic lupus erythematosus. Medicine. 2018 02; 46(2): 8492. https://doi.org/10.1016/j.mpmed.2017.11.010

14. Muro Y, Sugiura K, Akiyama M. Cutaneous Manifestations in Dermatomyositis: Key Clinical and Serological Features - a Comprehensive Review. Clinical Reviews in Allergy \& Immunology. 20150623 ; 51(3): 293-302. https://doi.org/10.1007/s12016-015-8496-5

15. Denton CP, Khanna D. Systemic sclerosis. The Lancet. 2017 Oct; 390(10103): 16851699.https://doi.org/10.1016/s0140-6736(17)30933-9

16. Pearson DR, Werth VP, Pappas-Taffer L. Systemic sclerosis: Current concepts of skin and systemic manifestations. Clinics in Dermatology. $2018 \quad 07 ; \quad 36(4)$ : 474.https://doi.org/10.1016/j.clindermatol.2018.04.004

17. Guiducci S, Bellando-Randone S, Matucci-Cerinic M. A New Way of Thinking about Systemic Sclerosis: The Opportunity for a Very Early Diagnosis. Israel Medical Association Journal [Internet]. 2016 [citado 2019 Mar 22]; 18(3-4), 141-143. Disponible en:https://www.ima.org.il/FilesUpload/IMAJ/0/193/96902.pdf

18. Bellando-Randone S, Matucci-Cerinic M. Very Early Systemic Sclerosis and Pre-systemic Sclerosis: Definition, Recognition, Clinical Relevance and Future Directions. Current Rheumatology Reports. 2017 09 18; 19: 65. https://doi.org/10.1007/s11926-017-0684-2 